\title{
Herstellung von Gewinden durch Gewindeschneiden in Gegengewichtsbohrungen von Großkurbelwellen
}

\author{
Norbert Miersch, Rainer Berghaus, Martin Lustig, Steffen Drechsler
}

Zusammenfassung

Das Thema beschäftigt sich mit dem Herstellen von Gewinden in Gegengewichtsflächen von Großkurbelwellen. Diese Publikation ist ein Teil der angewandten Forschung. Bei großen Gewindebohrungen ist das Gewindeschneiden produktiver als Gewindefräsen. Die Geschäftsführung der Gröditzer Kurbelwelle GmbH (GKW GmbH) möchte das Verfahren in die Produktion einführen. Der Herstellungsprozess muss dazu reproduzierbar und sicher und die Lebensdauer der Gewindebohrer bekannt sein.
Abstract

The theme deals with the production of threads in counterwights planes for large crankshafts. This publication is part of applied research. For large thread holes is thread cutting more productive than thread milling. The management of the company Gröditzer Kurbelwelle $\mathrm{GmbH}$ (GKW $\mathrm{GmbH}$ ) would like to implement this method in the production. The manufacturing process has to be reproducible and ensured. Therefore the threading tool's lifetime has to be well known.
Die TH Wildau [FH] hat im Drittmittelprojekt »Gewindeschneiden« im Team mit Projektleiter Prof. Dr.-Ing. Norbert Miersch und den Studenten Rainer Berghaus und Martin Lustig die Problematik des Gewindeschneidens wissenschaftlich bearbeitet und eine praktische, anwendungsorientierte Lösung erarbeitet. Die Ergebnisse wurden der GKW GmbH präsentiert und das Projekt zu einem positiven Abschluss gebracht.

\section{Einleitung und Motivation}

In der Firma Gröditzer Kurbelwelle Wildau GmbH (GKW GmbH) werden Großkurbelwellen im Längenbereich von $6 \mathrm{~m}$ bis $12 \mathrm{~m}$ hergestellt. Die mechanische Bearbeitung ist durch den Einsatz moderner, numerisch gesteuerter Werkzeugmaschinen, die zum größten Teil Sondermaschinen sind, geprägt. Zu nennen wären hier die GFM-Großkurbelwellenfräsmaschine zur Grundformgebung (Scheibenfräser mit Durchmessern von 1,60 m und 2,10 m), Drehfräsmaschinen für die Vor- und Fertigbearbeitung, Schmierlochbohrmaschinen (Einlippenbohren) sowie Dreh-, Fräs- und Schleifmaschinen.

Sehr produktiv sind die Drehfräsmaschinen, mit denen man in der Fertigbearbeitung die Grund- und Hublager einer Kurbelwelle durch Drehfräsen bearbeiten kann. Mit diesen Sondermaschinen kann auch eine
Vielzahl von Nebenformelementen hergestellt werden, wie beispielsweise Gewinde in Gegengewichtsflächen der Kurbelwellenwangen. Als Herstellungsverfahren wird das Gewindefräsen und das Gewindeschneiden angewandt.

Die Fertigungstechnologie wird bei Wiederholproduktion unter Berücksichtigung des aktuellen Technikstandes schrittweise angepasst (optimiert). Dabei werden progressive Werkzeuge eingesetzt. $\mathrm{Zu}$ nennen wären zum Beispiel Hartmetallstufenbohrer zum gleichzeitigen Bohren und Fasen der Kernbohrung eines Gewindes. Das An- und Vorbohren der Kernlochbohrung entfällt.

Die modernen, kostensparenden Technologien führen anfangs nicht immer zum gewünschten Erfolg (Einsparung der Grundzeit). So beträgt beim Gewindefräsen von Gewinden M 52x3 die Herstellungszeit der Gegengewichtsbohrungen für eine Kurbelwelle mit 24 Gewinden ca. 8 - 12 Stunden. Dabei kann die Qualität der Gewindebohrungen als gleichbleibend gut und ohne Ausschuss beurteilt werden. Für die gleichen Gewinde und gleiche Gewindeanzahl beträgt die Herstellungszeit durch den Einsatz des Gewindeschneidens nur die halbe Zeit, ca. 4 - 6 h. Bei den gefertigten Gewinden gibt es jedoch Qualitätsmängel. Der Gewindebohrer hat einen unkontrollierten Verschleiß sowie Beschädigungen (Ausbrüche der Schneide). Die dadurch ent- 
standenen fehlerhaften Gewindebohrungen können im schlimmsten Fall (worst case) den Ausschuss der gesamten Kurbelwelle zur Folge haben.

Ein weiteres Problem stellt der unsichere Gewindeherstellungsprozess in der Fertigung dar (nicht reproduzierbare und unkontrollierte Einflussgrößen). Aufgrund der höheren Produktivität des Gewindeschneidens gegenüber dem Gewindefräsen wurde durch die Geschäftsführung der GKW GmbH angedacht, dieses Verfahren wieder in die Fertigung aufzunehmen. Um sichere Aussagen bezüglich der Einsatzdauer von Gewindebohrern bezogen auf die Anzahl zu fertigender Gewindebohrungen zu erhalten, waren spezielle Zerspanungsversuche mit der Aufnahme prozessrelevanter Parameter erforderlich.

\section{Literaturrecherche}

Das Gewindeschneiden ist ein bewährtes spanendes Herstellungsverfahren, das über viele Jahre in der mechanischen Bearbeitung von Einzelteilen und der Montage von Baugruppen im Maschinenbau eingesetzt wird. Bezogen auf die bestimmten Einflussgrößen wie Art des Gewindes, Fertigungsverfahren, Werkzeugbeschichtung, Kühlschmierung, Temperatur, Schnittkraft, Drehmoment, Schneidstoff, Werkstückwerkstoff und Schnittgeschwindigkeit gibt es viele Neuerungen (Optimierungsansätze), die sich im Wesentlichen auf kleinere Gewinde wie M10 bis M20 beziehen.

Um den Gewindeschneidprozess sicherer zu gestalten, werden im Verbund von Werkzeugmaschine/Werkzeugaufnahme Sensoren zur Prozesskontrolle eingesetzt (beispielweise Temperatur-, Kraft- und Drehmomentsensoren). Moderne Werkzeugaufnahmen werden vom Werkzeughersteller heutzutage mit einem Drehmomentausgleich und/oder Längenausgleich ausgestattet.

Auch die Werkzeugmaschinenhersteller bieten eine integrierte Sensorik zur Prozesskontrolle des Zerspanungsprozesses an (z. B. Anzeige der Zerspanungsleistung, Werkzeugbruchkontrolle).

In [1] wird beschrieben, dass viele Hersteller das Zirkularfräsen dem noch weit verbreiteten Gewindebohren vorziehen. Es werden hier noch weitere Gewindeherstellungsverfahren wie das Bohrgewindefräsen und Sonderkombinationen genannt. Dabei wird darauf hingewiesen, dass nur ein qualifizierter Vergleich für den konkreten Anwendungsfall eine wirtschaftliche Lösung hervorbringt.
In Tabelle 1 sind die wichtigsten Bemühungen (Auszug) bezogen auf das Gewindeschneiden zusammengestellt.

\section{Grundlagen des Kernlochbohrens}

\section{Kernlochdurchmesser und Toleranzen}

Um die Herstellung eines Innengewindes zu ermöglichen, muss eine Grundbohrung mit einem Kernlochdurchmesser erzeugt werden. Nach [13] wird der Kernlochdurchmesser des Innengewindes mit

$\mathrm{D}_{1}=\mathrm{d}-1,0825 \approx \mathrm{P}$

$\mathrm{D}_{1}$ - Kerndurchmesser des Innengewindes [mm]

d - Gewindenenndurchmesser [mm]

P - Steigung des Gewindes [mm]

berechnet. In [14] wird darauf hingewiesen, dass bei zähen Werkstoffen durch Gratbildung vor dem Schneiden erhöhte Werkzeugbruchgefahr besteht. Hierzu sollte der Lochdurchmesser für die Grundbohrung etwas größer sein als der Kerndurchmesser.

Nach [14] wird dazu folgende Formel angegeben:

$\mathrm{d}_{\mathrm{K}} \approx \mathrm{d}-1,1 \cdot \mathrm{P}_{\mathrm{w}}$

$\mathrm{d}_{\mathrm{K}}$ - Lochdurchmesser [mm]

$\mathrm{d}$ - Gewindenenndurchmesser [mm]

$\mathrm{P}_{\mathrm{w}}$ - Steigung des Gewindes [mm]

Um die Berechnung der Kernbohrung zu verdeutlichen, wird folgendes Berechnungsbeispiel angegeben.

\section{Beispiel:}

Für ein Gewinde M52x3 mit Toleranzklasse $6 \mathrm{H}$ soll in einem Vergütungsstahl aus 42CrMoNi4V [A1] eine Kernbohrung erzeugt werden.

Gewählt wird hierzu ein Wendeschneidplattenbohrer der Firma Stellram [18] mit langem Weldon [A2] Schaft nach Artikel S6300W440R für einen Kerndurchmesser $D_{1}=49 \mathrm{~mm}$. Auswahl der Wendeschneidplatte Form P für legierte Stähle.

\section{Lösung:}

Für die Toleranzklasse des Gewindes $6 \mathrm{H}$ für 52x3 können aus den Toleranztabellen [15] folgende Größt- und Kleinstmaße entnommen werden:

$\mathrm{D}_{1 \mathrm{~min}}=48,752 \mathrm{~mm}$

$\mathrm{D}_{1 \max }=49,252 \mathrm{~mm}$

Nach Formel (1) ergibt das einen Kerndurchmesser $D_{1}$ von mindestens 48,752 mm. Wobei nach Formel (2) ein Lochdurchmesser von mindestens $\mathrm{dk} \approx 48,7 \mathrm{~mm}$ empfohlen wird. Diese Werte stellen die unteren Toleranz- 
Einflussgröße, Quelle Jahr Beschreibung Maßnahme
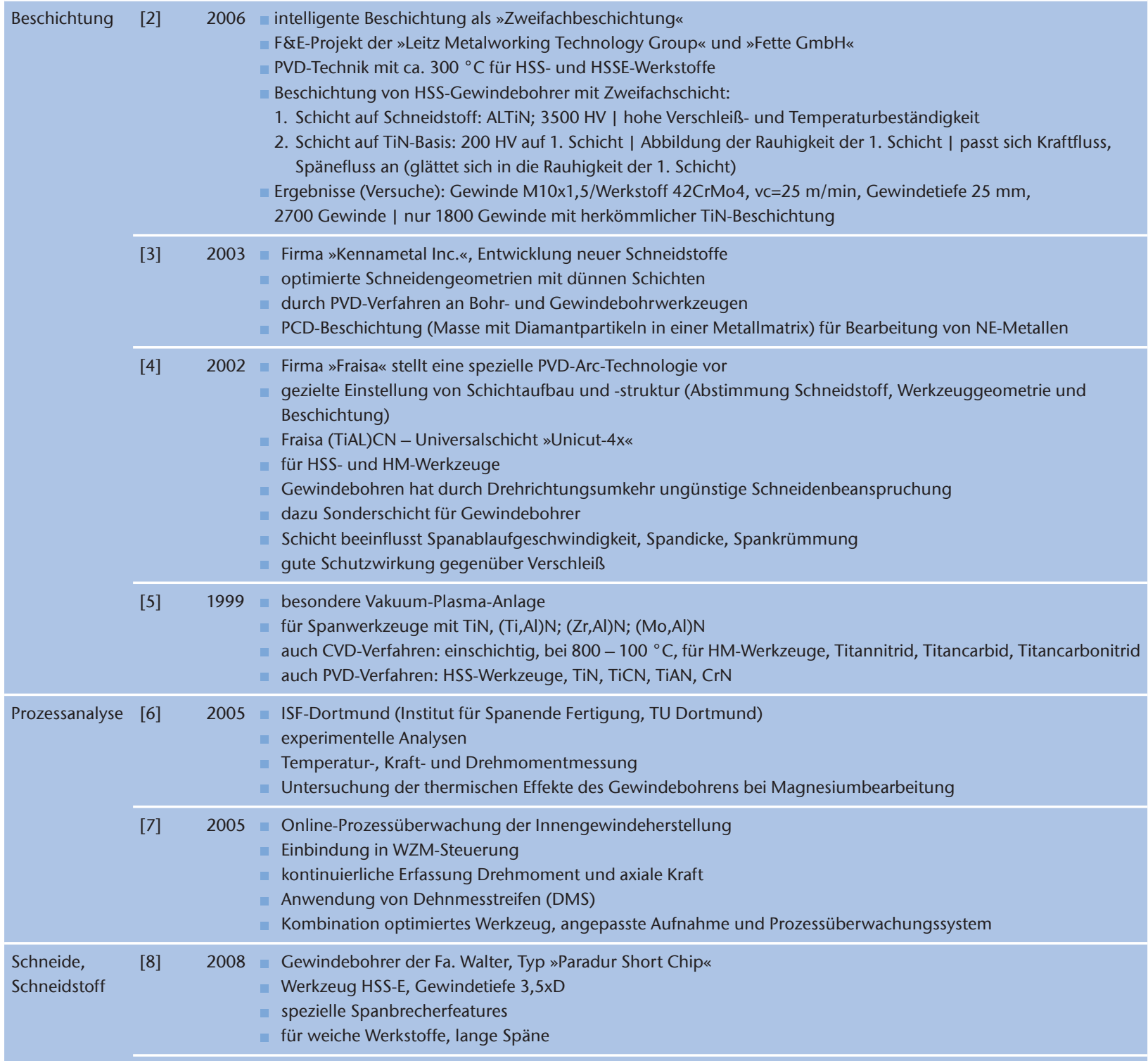

[9] 2004 Schumacher GmbH \& Co. KG, Remscheid

- neuer Vollhartmetallgewindebohrer

Werkstoffe: S235JR(St37-2), S355J2G3(St52-3N), V2A, EC80, C45, - vc $=50-70 \mathrm{~m} / \mathrm{min}$, Synchronspindel

Tiefe 2D - 2,5 D, 80 bar Innenkühlung, 6\%ige Emulsion

\begin{tabular}{|c|c|c|c|}
\hline \multirow[t]{3}{*}{$\begin{array}{l}\text { Kühl- } \\
\text { schmierung }\end{array}$} & [10] & 2005 & $\begin{array}{l}\text { Kosteneinsparung durch Umstellung der Schmierung } \\
\text { - Minimalmengenschmierung, Trockenbearbeitung } \\
\text { - Grundlochgewindebohren mit VHM und HSS-E-PM } \\
\text { - Anpassung der Schneidengeometrie (Freiwinkel erhöhen) } \\
\text { Multilayer-Hartstoffbeschichtung für HSS-E mit TiAIN } \\
\text { strömungstechnische Anpassung der Kühlschmierstoffkanäle }\end{array}$ \\
\hline & [11] & 2002 & $\begin{array}{l}\text { - Patent »Unicut-Duplex-Spraying-System« } \\
\text { " neuartige Minimalmengenschmierung (MMS) } \\
\text { " Kombination zweier nicht mischbarer Fluide } \\
\text { " gesonderte Kühlkanäle, Kühlung mit Wasseranteil } \\
\text { " Funktionen Kühlung und Schmierung } \\
\text { " Anwendung bei Gewindebohren } \\
\text { " für Stähle (hochfest, austenitisch), Ni, } \mathrm{Ti} \text { - Legierungen }\end{array}$ \\
\hline & [12] & 2001 & $\begin{array}{l}\text { - Anwendung biologisch abbaubarer Kühlschmierstoffe und Minimalmengenschmierung } \\
\text { positive Ergebnisse bei Gewindebohren von Superlegierungen ( } \mathrm{Cr}, \mathrm{Ni} \text { - Basis), Alu-Legierungen } \\
\text { Alternative zur konventionellen Schmierung }\end{array}$ \\
\hline
\end{tabular}

Tab. 1: Auszüge aus der internationalen Literaturrecherche 
grenzen für die Abmaße der Kernlochbohrung nach $6 \mathrm{H}$ dar [15].

In [15] wird der Vorbohrdurchmesser nach DIN 336 angegeben. Er ist abhängig vom Verhalten des Materials und den schneidtechnischen Betriebsverhältnissen. Es wird in [15] darauf hingewiesen, dass andere Vorbohrdurchmesser, besonders bei Serienfertigung, individuell zu ermitteln sind. Dabei ist eine wirtschaftliche Gewindefertigung bei Ausnutzung der oberen Toleranzgrenze besser gegeben.

\section{Fehlerursachen, Fehlerbeseitigung}

In [15] wird in einer übersichtlichen Tabelle folgende Fehlerbetrachtung zu den Kernlochbohrungen zusammenfassend dargestellt.

\section{Werkzeuge zum Kernlochbohren in der GKW GmbH} Zur Herstellung der Grundbohrungen in den Gegengewichtsflächen der Kurbelwellenwangen werden Wendeschneidplattenbohrer verwendet.

Hierzu wird die Hauptspindel an der Drehfräsmaschine in den Koordinatenachsen Y,Z in Bohrposition gefahren (X-Achse ist der Verfahrweg für die Bohrungstiefe). Die Bohrung wird ohne An- und Vorbohren hergestellt.

Die Gewindefase kann durch eine zusätzliche Stufe im Bohrer gleichzeitig mit erzeugt werden. Abb. 1 zeigt beispielhaft einen in der Fa. GKW GmbH angewandten Wendeplattenbohrer.

Zur Herstellung einer Kernlochbohrung $D_{1}=49 \mathrm{~mm}$ für ein Gewinde M52x3 werden in der GKW GmbH folgende technologischen Arbeitswerte vorgegeben:

$\mathrm{n}_{\mathrm{w}}=550 \mathrm{U} / \mathrm{min}$ (Bohrerdrehzahl)

$\mathrm{v}_{\mathrm{c}}=85 \mathrm{~m} / \mathrm{min}$ (Schnittgeschwindigkeit)

$\mathrm{F}=200 \mathrm{~mm} / \mathrm{min}$ (Vorschubgeschwindigkeit)

$\mathrm{z}=2$ (Anzahl der Schneiden)

$\mathrm{f}_{\mathrm{z}}=0,18 \mathrm{~mm} / \mathrm{U}$ (Vorschub pro Zahn)

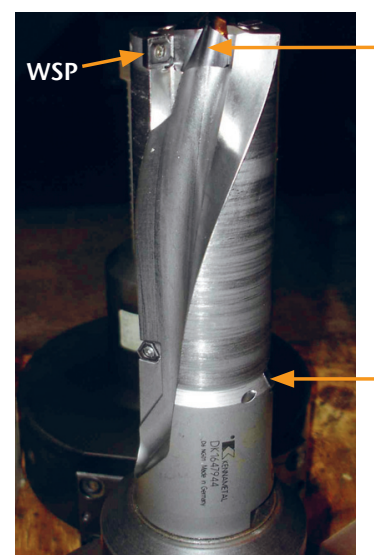

- Piloteneinsatz HM

WSP und Absatz für Fase

Abb. 1: Wendeplattenbohrer der Firma KENNAMETAL (WSP = Wendeschneidplatte; $\mathrm{HM}=$ Hartmetall)

\section{Auswahl der Werkzeugaufnahme}

Für das Gewindeschneiden müssen Gewindebohrer und Werkzeugaufnahme (Schneidfutter) aufeinander abgestimmt sein [15]. Da die in der GKW GmbH eingesetzte Drehfräsmaschine zur Herstellung der Gewindebohrungen keine Synchronspindel besitzt, muss aufgrund der Fehlertoleranz des benutzten Vorschubantriebes an der WZM ein axialer Toleranzausgleich durch die Werkzeugaufnahme sichergestellt werden. Außerdem sollte das Schneidfutter ein maximal vertretbares Gewindeschneidmoment begrenzen. Dafür gibt es Überlastkupplungen an bestimmten Schneidfuttern. Die Einstellung erfolgt durch die Mitarbeiter der Werkzeugvoreinstellung in der GKW. Die Überlastkupplung ermöglicht eine Begrenzung des Drehmomentes für das Gewindeschneiden. Das einzustellende Grenzdrehmoment kann der Bedienungsanleitung [19] entnommen werden. Es ist von der jeweiligen Gewindegröße abhängig.

Nach Recherchen in [16] wird das Gewindeschneidfutter zum Maschinentyp abgestimmt. Der Gewindebohrer wird durch den Vierkant formschlüssig in das Futter aufgenommen. So wurde von der Fa. EMUGE zum Schneiden des Gewindes M52x3 das Gewindeschneidfutter HF 20/IKZ empfohlen. Aufgrund der Möglichkeit der inneren Kühlmittelzufuhr (IKZ) besitzt das Ausgleichsfutter nur die Funktion des axialen Längenausgleichs in Druck- und Zugrichtung (Abb. 2). Weitere mögliche Ausgleichsfunktion für Gewindeschneidfutter werden in [15] und [16] beschrieben. Der Längenausgleich kompensiert Differenzen zwischen Spindelvorschub und Gewindebohrersteigung (Abb. 2).

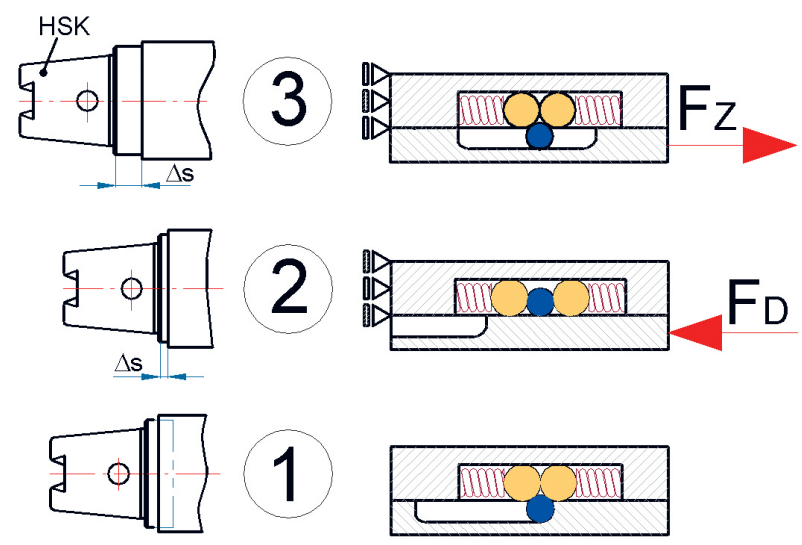

Abb 2: Funktion des Längenausgleichs beim Gewindeschneidfutter HF 20/IKZ (1 Schneidvorgang ohne Längenausgleich; 2 Schneidvorgang mit Längenausgleich in Druckrichtung; 3 Schneidvorgang mit Längenausgleich in Zugrichtung; FD = Druckkraft durch Toleranzabweichung bzw. Überlastung; Fz = Zugkraft durch Toleranzabweichung; HSK = Hohlschaftkegelaufnahme (Maschinenspindelaufnahme)) 


\section{Gewindebohren}

Benennungen und Definitionen am Gewindebohrer Wichtige Kenngrößen und Winkel werden in Abb. 3 dargestellt.

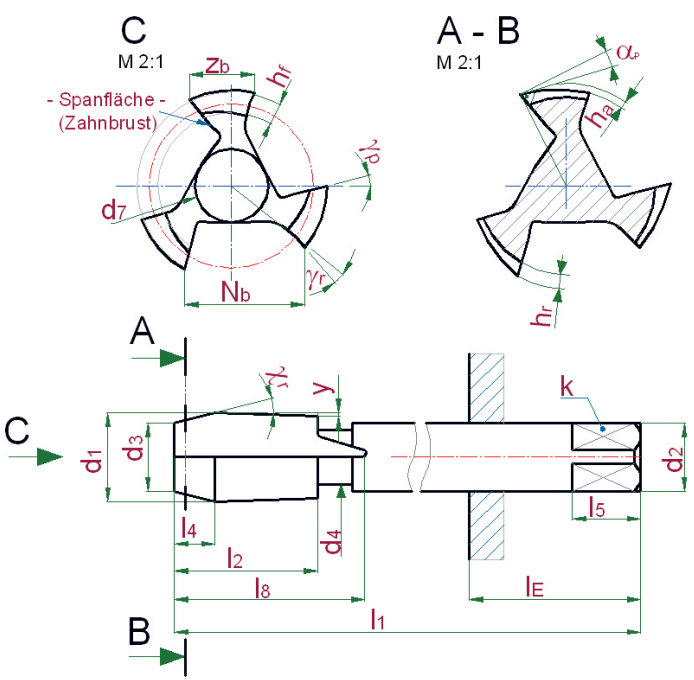

Abb. 3: Kenngrößen und Winkel am Gewindebohrer in Anlehnung an [15]

$\mathrm{d}_{1}=$ Gewindenenn $\varnothing ; \mathrm{d}_{2}=$ Schaft $\varnothing ; \mathrm{d}_{3}=$ Anschnitt $\varnothing ; \mathrm{d}_{4}=$ Hals $\varnothing ;$

$\mathrm{d}_{7}=$ Seelen $\varnothing ; \mathrm{l}_{1}=$ Gesamtlänge; $\mathrm{I}_{2}=$ Gewindelänge; $\mathrm{I}_{4}=$ Anschnittlänge;

$\mathrm{I}_{5}=$ Vierkantlänge; $\mathrm{I}_{8}=$ Nutenlänge; $\mathrm{I}_{\mathrm{E}}=$ Einspannlänge; $\chi_{\mathrm{r}}=$ Einstellwinkel; $\gamma_{\mathrm{p}}=$ Spanwinkel (erster voller Zahn); $\gamma_{\mathrm{r}}=$ Rückenwinkel; $\alpha_{\mathrm{p}}=$ Anschnittfreiwinkel; $y=1 / 2$ Durchmesserverjüngung; $k=$ Vierkantmaß; $z_{b}=$ Zahnbreite; $N_{b}=$ Nutenbreite; $h_{a}=$ Anschnitthinterschliff (auf Zahnbreite); $h_{\mathrm{r}}=$ Anschnitthinterschliff (pro Teilung); $h_{\mathrm{f}}=$ Flankenhinterschliff (auf Zahnbreite)

\section{Anschnittsformen}

Jede Gewindebohrerart hat eine definierte Anschnittsform. Die Anschnittsformen und Anschnittslängen sind nach DIN 2197 festgelegt (siehe auch [15]).

\section{Verfahrensanalyse}

Der Drehmomentverlauf beim Gewindeschneidvorgang kann in Zeitabschnitte eingeteilt werden. Die den Zeitabschnitten zugeordneten Drehmomente werden in Abb. 4 dargestellt.

Schnittgeschwindigkeit und Kühlschmierung

Bei den Schnittgeschwindigkeiten wird nach [15] für beschichtete Gewindebohrer (HSS-E mit TiN) bezogen auf Vergütungsstähle (z. B. 42CrMo4V) ein Geschwindigkeitsbereich von $\mathrm{v}_{c}(2 \ldots 20) \mathrm{m} / \mathrm{min}$ bei $\mathrm{R}_{\mathrm{e}}=900-1100$ $\mathrm{N} / \mathrm{mm}^{2}$ empfohlen. Als Kühlschmierstoff wird ein speziell von EMUGE entwickeltes Gewindeschneidöl für vertikales Gewindebohren und Schneidpaste für horizontales Gewindebohren vorgeschlagen. Schneidöl und Schneidpaste werden in Abhängigkeit vom Werkstückwerkstoff aus einer Tabelle in [16] ausgewählt. Bei

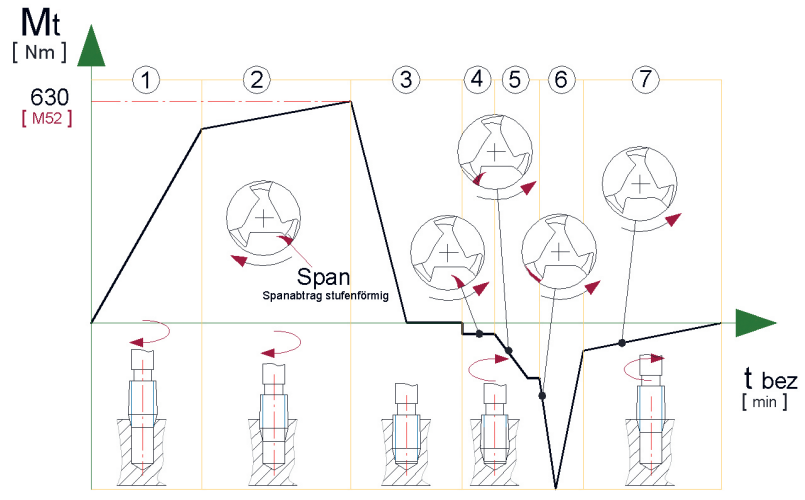

Abb. 4: Drehmomentverlauf beim Gewindeschneiden in einer Grundbohrung $\left(\mathrm{M}_{\mathrm{t}}=\right.$ Drehmoment in $[\mathrm{Nm}]$; $\mathrm{t}_{\text {bez }}=$ Zeit bezogen auf das Schneiden eines Gewindes in [min])

Zeitstufe (Beschreibung): 1 (Anschneiden); 2 (Gewindeschneiden voller Eingriff); 3 (Abbremsen bis Stillstand); 4 (Rücklauf - bis Kontakt Span an Spanstegrücken); 5 (Rücklauf - Abscheren des Spanes); 6 (Rücklauf Zurückformen der Scherstelle des Spanes); 7 (Rücklauf - Gleitreibung)

ausreichendem Druck, um die Kühlung und Späneentsorgung zu gewährleisten (ca. 50 - 80 bar), können auch Werkzeuge mit innerer Kühlmittelzufuhr (IKZ) unter Verwendung von Bohremulsion eingesetzt werden. Andere Literaturquellen wie [13], [14], [17] geben weitere (abweichende) Schnittgeschwindigkeitsbereiche an. Dazu werden noch Kühlschmiermittel Rüböl oder Schneidöl geschwefelt vorgeschlagen. Der Anwender muss also die zweckmäßigen Schnittgeschwindigkeiten selbst festlegen oder durch Versuche ermitteln. Die Auswahl der zweckmäßigen Schnittgeschwindigkeit hängt im Wesentlichen von den Einflußgrößen

- Werkstückwerkstoff,

- Kühlschmierung,

- Gewindetiefe,

- Gewindedurchmesser,

- Werkstückspannung und

- Werkzeugmaschine ab.

Zur Festlegung wird nach Abb. 5 folgende Hilfestellung gegeben:

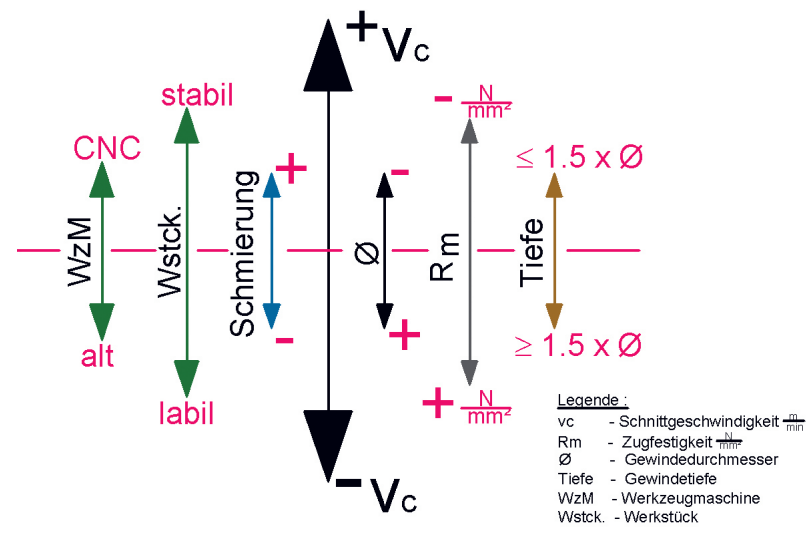

Abb. 5: Hilfe zur Auswahl der Schnittgeschwindigkeit $v_{c}[\mathrm{~m} / \mathrm{min}]$ 


\section{Gewindebohrer}

Entsprechend dem zu bearbeitenden Werkstoff und der Gewindegröße, der Art der Grundbohrung (Grundbohrung, Durchgangsbohrung) und der Tiefe der Gewindebohrung können aus [15] und [16] der Typ des Gewindebohrers, die Bauform und die entsprechenden Merkmale ausgewählt werden. Es werden in der GKW GmbH im Wesentlichen zwei Typen von Gewindebohrern der Fa. EMUGE [16] für das Herstellen von Gegengewichtsbohrungen verwendet (siehe Tabelle 2).

\section{Fehleranalyse beim Gewindeschneiden}

Beim Gewindeschneiden wirken mehrere Einfluss- größen einzeln oder zusammenhängend. Zusammenfassend können die Einflussgrößen den Kategorien

- Werkzeug,

- Werkzeugmaschine,

- Werkstück,

- Hilfsstoff

zugeordnet werden. Die Erfahrung eines qualifizierten Maschinenbedieners wird hier vorausgesetzt. Systematisiert man grundlegende Gewindefehler, die aus diesen Einflussgrößen resultieren, so können Herstellungsfehler bezogen auf das Gewindeschneiden, mögliche Fehlerursachen und Vorschläge zu deren Beseitigung in Tabelle 3 zusammengefasst (siehe [15]) werden.

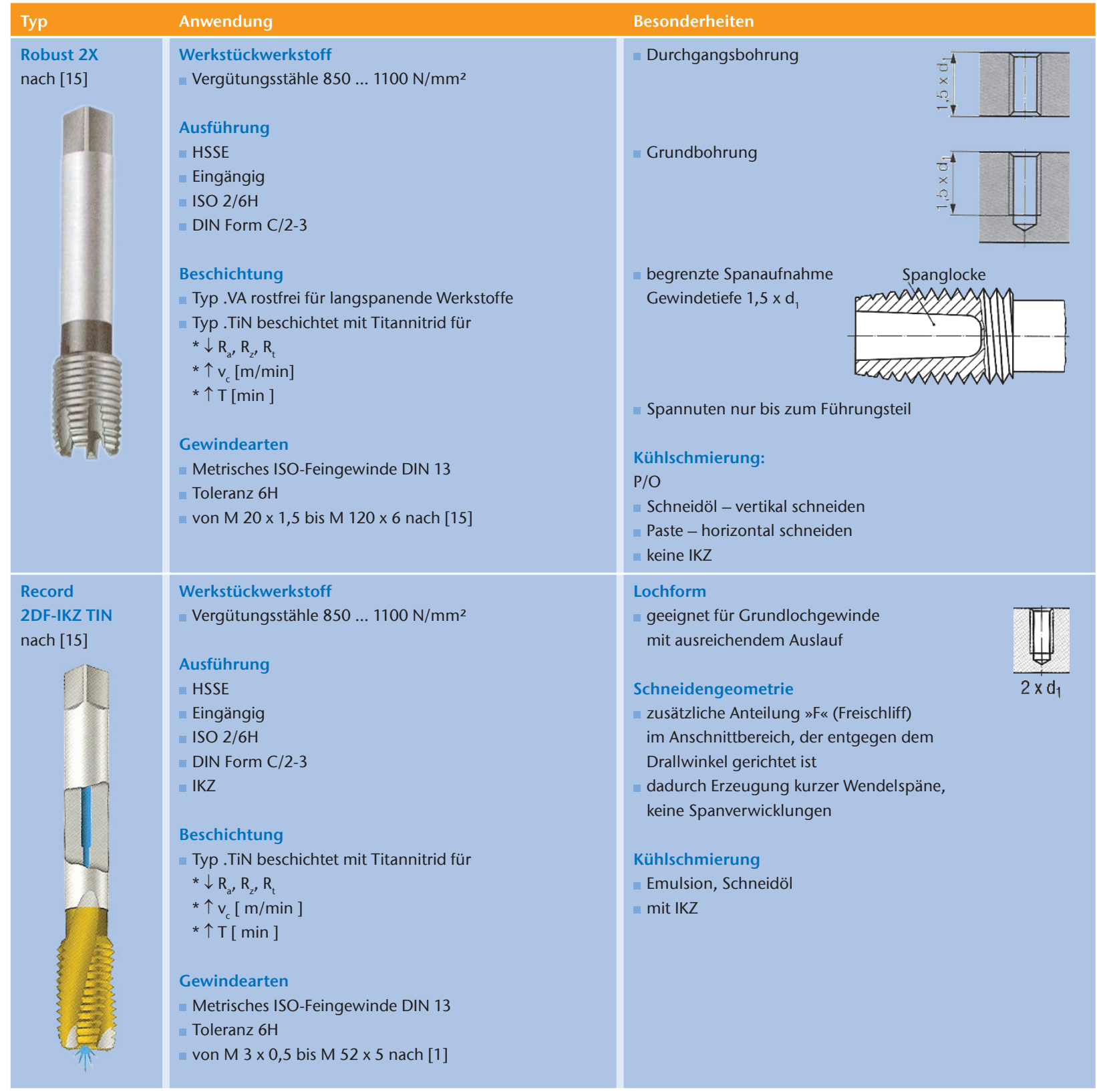

Tab. 2: Gewindebohrertypen nach [15], [16] für den Einsatz in der GKW GmbH ( $\mathrm{d}_{1}=$ Gewindenenndurchmesser [mm]; $\mathrm{R}_{\mathrm{m}}=\mathrm{Zugfestigkeit} \mathrm{[N/mm}{ }^{2}$; $\mathrm{v}_{\mathrm{c}}=$ Schnittgeschwindigkeit [m/min]; $\mathrm{T}=$ Standzeit [min]; IKZ = Innere Kühlmittelzufuhr; C/2-3 = Anschnittsform C mit $2-3$ Gängen im Anschnitt) 


\begin{tabular}{|c|c|c|c|c|}
\hline \multirow{4}{*}{$\begin{array}{l}\text { Fehler } \\
\text { (Gewinde) } \\
\text { zu groß }\end{array}$} & \multicolumn{2}{|l|}{ Ursache } & \multicolumn{2}{|c|}{ Beseitigung } \\
\hline & Werkzeug & $\begin{array}{l}\text { falsche Auswahl } \\
\text { Geometrie der Schneide ungeeignet } \\
\text { hohe Verschleißmarkenbreite } \\
\text { schlechte Spanabfuhr }\end{array}$ & Werkzeug & $\begin{array}{l}\text { Auswahl korrigieren nach Materialgruppe, Kühlschmierung, } \\
\text { Spannuten, Anschnitt, Bohrungsart } \\
\text { arbeitsscharfes Werkzeug wählen } \\
\text { geeignetes Ausgleichsfutter wählen }\end{array}$ \\
\hline & $\begin{array}{l}\text { Werkzeug- } \\
\text { maschine }\end{array}$ & $\begin{array}{l}\text { zu hohe Vorschubtoleranz der WZM- } \\
\text { Spindel bei Gewindeschneidfutter } \\
\text { mit Längenausgleich } \\
\text { Schnittgeschwindigkeit zu hoch }\end{array}$ & $\begin{array}{l}\text { Werkzeug- } \\
\text { maschine }\end{array}$ & $\begin{array}{l}\text { — Schnittgeschwindigkeit verringern } \\
\text { — andere Werkzeugmaschine wählen }\end{array}$ \\
\hline & Werkstück & $\begin{array}{l}\text { zu kleine Kernlochbohrung } \\
\text { falsche Werkstückspannung }\end{array}$ & Werkstück & $\begin{array}{l}\text { - Kernlochdurchmesser nach DIN } 13 \text { und DIN ISO 965-1 fertigen } \\
\text { " Werkstückspannung ändern }\end{array}$ \\
\hline \multirow[t]{3}{*}{ Ausreißen } & Werkzeug & $\begin{array}{l}\text { falsche Auswahl } \\
\text { hohe Verschleißmarkenbreite } \\
\text { verschlissener Gewindebohrer } \\
\text { veränderliche Werkstoffeigenschaften } \\
\text { schlechte Spanabfuhr, drücken beim } \\
\text { Herausdrehen }\end{array}$ & Werkzeug & $\begin{array}{l}\text { Auswahl korrigieren nach Materialgruppe, Kühlschmierung, } \\
\text { Spannuten, Anschnitt, Bohrungsart } \\
\text { arbeitsscharfes Werkzeug wählen } \\
\text { geeignetes Ausgleichsfutter wählen } \\
\text { Auswahl nach Werkstofffestigkeit korrigieren }\end{array}$ \\
\hline & $\begin{array}{l}\text { Werkzeug- } \\
\text { maschine }\end{array}$ & $\begin{array}{l}\text { zu hohe Vorschubtoleranz der WZM- } \\
\text { Spindel bei Gewindeschneidfutter } \\
\text { mit Längenausgleich } \\
\text { Schnittgeschwindigkeit zu hoch }\end{array}$ & $\begin{array}{l}\text { Werkzeug- } \\
\text { maschine }\end{array}$ & $\begin{array}{l}\text { Schnittgeschwindigkeit verringern } \\
\text { andere Werkzeugmaschine wählen }\end{array}$ \\
\hline & Hilfsstoff & ص Versagen der Kühlschmierung & Hilfsstoff & $\begin{array}{l}\text { Gewindebohrer mit Möglichkeit einer ausreichenden Kühlschmier- } \\
\text { stoffzufuhr wählen } \\
\text { Kühlschmierstoffart ändern }\end{array}$ \\
\hline \multirow[t]{2}{*}{$\begin{array}{l}\text { axial ver- } \\
\text { schnitten }\end{array}$} & Werkzeug & $\begin{array}{l}\text { schlechte Spanabfuhr, drücken beim } \\
\text { Herausdrehen }\end{array}$ & Werkzeug & $\begin{array}{l}\text { Auswahl korrigieren nach Spannuten, Anschnitt, Bohrungsart } \\
\text { geeignetes Ausgleichsfutter wählen } \\
\text { = Überlastkupplung der Aufnahme korrekt einstellen }\end{array}$ \\
\hline & $\begin{array}{l}\text { Werkzeug- } \\
\text { maschine }\end{array}$ & geringe Genauigkeit der Vorschubachse & $\begin{array}{l}\text { Werkzeug- } \\
\text { maschine }\end{array}$ & = andere Werkzeugmaschine wählen \\
\hline zu eng & Werkzeug & $\begin{array}{l}\text { falsche Auswahl } \\
\text { Toleranzangabe auf dem Gewindebohrer } \\
\text { ist nicht identisch mit der Toleranzangabe } \\
\text { in der Zeichnung oder der Gewindelehre }\end{array}$ & Werkzeug & घ Gewindebohrer mit entsprechender Toleranz verwenden \\
\hline \multirow[t]{2}{*}{ konisch } & Werkzeug & $\begin{array}{l}\text { Gewindebohrer schneidet nicht steigungs- } \\
\text { genau (Gewinde-Gut-Lehrdorn lässt sich } \\
\text { nicht vollständig einschrauben) }\end{array}$ & Werkzeug & $\begin{array}{l}\text { - übermäßige Axialkräfte während des Schneidvorganges vermeiden } \\
\text { - Gewindeschneidfutter mit Längenausgleich verwenden }\end{array}$ \\
\hline & & & $\begin{array}{l}\text { Werkzeug- } \\
\text { maschine }\end{array}$ & $\begin{array}{l}\text { übermäßige Axialkräfte während des Schneidvorganges vermeiden } \\
\text { Werkzeugmaschine mit Synchronspindel }\end{array}$ \\
\hline \multirow[t]{3}{*}{ Vorweite } & Werkzeug & $\begin{array}{l}\text { falscher Anschnittsdruck } \\
\text { Winkel oder Positionsfehler der } \\
\text { Gewinde-Kernlochbohrung }\end{array}$ & Werkzeug & = Gewindeschneidfutter mit Längenausgleich einsetzen \\
\hline & & & $\begin{array}{l}\text { Werkzeug- } \\
\text { maschine }\end{array}$ & $\begin{array}{l}\text { übermäßige Axialkräfte während des Schneidvorganges vermeiden } \\
\text { Werkzeugmaschine mit Synchronspindel verwenden und mit } \\
\text { Zwangsvorschub arbeiten }\end{array}$ \\
\hline & Werkstück & $\begin{array}{l}\text { Wechsel der technologischen Basis } \\
\text { Toleranzabweichung durch falsche } \\
\text { Lagebestimmung }\end{array}$ & Werkstück & $\begin{array}{l}\text { Kernlochdurchmesser nach DIN } 13 \text { und DIN ISO 965-1 fertigen } \\
\text { = Werkstückspannung ändern }\end{array}$ \\
\hline \multirow[t]{3}{*}{$\begin{array}{l}\text { schlechte } \\
\text { Ober- } \\
\text { fläche }\end{array}$} & Werkzeug & $\begin{array}{l}\text { falsche Auswahl } \\
\text { hohe Verschleißmarkenbreite } \\
\text { verschlissener Gewindebohrer } \\
\text { schlechte Spanabfuhr }\end{array}$ & Werkzeug & $\begin{array}{l}\text { Auswahl korrigieren nach Materialgruppe, Kühlschmierung, } \\
\text { Spannuten, Anschnitt, Bohrungsart } \\
\text { arbeitsscharfes Werkzeug wählen } \\
\text { scharfen Kernbohrer verwenden }\end{array}$ \\
\hline & Werkstück & —schlechte Oberfläche der Kernbohrung & $\begin{array}{l}\text { Werkzeug- } \\
\text { maschine }\end{array}$ & Schnittgeschwindigkeit verringern \\
\hline & Hilfsstoff & Versagen der Kühlschmierung & Hilfsstoff & $\begin{array}{l}\text { Gewindebohrer mit Möglichkeit einer ausreichenden Kühlschmier- } \\
\text { stoffzufuhr wählen } \\
\text { Kühlschmierstoffart ändern: Schneidfett bei waagerechter } \\
\text { Bohrung, Schneidöl bei senkrechter Bohrung } \\
\text { für ausreichend Druck und Fördermenge bei Bohremulsion sorgen } \\
\text { ausreichender Fettgehalt bei Emulsion }\end{array}$ \\
\hline \multirow[t]{2}{*}{ Bruch } & Werkzeug & $\begin{array}{l}\text { falsche Auswahl } \\
\text { hohe Verschleißmarkenbreite } \\
\text { verschlissener Gewindebohrer } \\
\text { schlechte Spanabfuhr }\end{array}$ & Werkzeug & $\begin{array}{l}\text { Auswahl korrigieren nach Materialgruppe, Kühlschmierung, } \\
\text { Spannuten, Anschnitt, Bohrungsart } \\
\text { arbeitsscharfes Werkzeug wählen } \\
\text { geeignetes Ausgleichsfutter wählen }\end{array}$ \\
\hline & Werkstück & $\begin{array}{l}\text { Spanverklemmungen, Spänestau } \\
\text { Gewindekernlochbohrung zu klein } \\
\text { Auflaufen des Gewindekernlochbohrers } \\
\text { auf Kernlochgrund } \\
\text { Winkel bzw. Positionsfehler der Bohrung }\end{array}$ & Werkstück & $\begin{array}{l}\text { Kernlochdurchmesser nach DIN } 13 \text { und DIN ISO 965-1 fertigen } \\
\text { - Werkstückspannung ändern }\end{array}$ \\
\hline
\end{tabular}

Tab 3: Mögliche Fehler beim Gewindeschneiden mit Maschinengewindebohrer $\left(v_{c}=\right.$ Schnittgeschwindigkeit $[\mathrm{m} / \mathrm{min}]$; WZM = Werkzeugmaschine $)$ 


\section{Zusammenfassung und Ausblick}

Das Gewindeschneiden in Metall ist ein bewährtes und altes Herstellungsverfahren. In [20] (1940) wird das Verfahren bereits ausreichend erläutert. Aufgrund der vielfältigen Einflussgrößen (multiple Einflüsse) auf Werkstück und Werkzeug ist das Gewindeschneiden großer Bohrungen (z. B. M52x3 in 42CrMo4V) bei seiner Anwendung in der Großkurbelwellenfertigung noch zu unsicher. Der Ausschuss eines Gewindes kann im schlimmsten Fall zum Ausschuss der gesamten Kurbelwelle führen.

Aufgrund der hohen Anforderungen der Motorenhersteller werden an eine Kurbelwelle immer höhere Qualitätsanforderungen gestellt. Besonders die Gewinde zur Montage der Gegengewichte müssen den immer größer werden Massekräften standhalten und dürfen keine Fehler aufweisen.

Durch die wissenschaftliche Aufarbeitung der Problematik, vor allem auch unter Berücksichtigung neuester wissenschaftlicher Erkenntnisse wurde in diesem 1. Teil auf den Stand der Technik eingegangen. Somit kann diese Veröffentlichung als praxisorientierte Ergänzung des Unterrichtsstoffes zum Gewindeschneiden angesehen werden. Bei der Betrachtung wurden bereits folgende Eingrenzungen berücksichtigt:

- Großkurbelwellen aus Vergütungsstahl 42CrMo4V, 42CrMoNi4V, 34CrNiMo6;

- Herstellung der Bohrungen zur Montage der Gegengewichte an der Kurbelwelle;

- spezielle Werkzeugmaschinen, Werkzeuge und Bedingungen aus der GKW GmbH.

Die internationale Forschung versucht das Verfahren vor allem in folgenden Schwerpunkten zu optimieren:

- Schneidstoffe;

- Schneidgeometrie;

- Beschichtung der Schneide;

- Erfassung und Auswertung von Prozessparametern;

- Kühlschmierung und Späneableitung.

Ein Forschungsteam der TH Wildau (Teamleiter Prof. Dr.-Ing. Norbert Miersch) bearbeitete dieses Thema. Es wurden neben der Recherche zum Stand der Technik umfangreiche Zerspanungsversuche durchgeführt.

\section{Kurzzeichenverzeichnis}

\begin{tabular}{|c|c|c|}
\hline Zeichen & Beschreibung & Einheit \\
\hline$\gamma_{p}$ & Spanwinkel (erster voller Zahn)/Gewindebohrer & $\circ$ \\
\hline$\alpha_{p}$ & Anschnittfreiwinkel/Gewindebohrer & $\circ$ \\
\hline$\chi_{r}$ & Einstellwinkel/Gewindebohrer & $\circ$ \\
\hline$\gamma_{\mathrm{r}}$ & Rückenwinkel/Gewindebohrer & $\circ$ \\
\hline C & Anschnittsform C/Gewindebohrer & \\
\hline CNC & $\begin{array}{l}\text { Numerisch gesteuerte Werkzeugmaschine } \\
\text { (computational numerical control) }\end{array}$ & \\
\hline d & Gewindenenndurchmesser & $\mathrm{mm}$ \\
\hline$D_{1}$ & $\begin{array}{l}\text { Durchmesser der Kernlochbohrung } \\
\text { nach DIN } 336\end{array}$ & $\mathrm{~mm}$ \\
\hline$d_{1}$ & Gewindenenndurchmesser & $\mathrm{mm}$ \\
\hline $\mathrm{D}_{1 \max }$ & Größtes Abmaß der Kernlochbohrung & $\mathrm{mm}$ \\
\hline $\mathrm{D}_{1 \min }$ & Kleinstes Abmaß der Kernlochbohrung & $\mathrm{mm}$ \\
\hline$d_{2}$ & Schaftdurchmesser/Gewindebohrer & $\mathrm{mm}$ \\
\hline$d_{3}$ & Anschnittdurchmesser/Gewindebohrer & $\mathrm{mm}$ \\
\hline$d_{4}$ & Halsdurchmesser/Gewindebohrer & $\mathrm{mm}$ \\
\hline$d_{7}$ & Seelendurchmesser/Gewindebohrer & $\mathrm{mm}$ \\
\hline$d_{k}$ & Lochdurchmesser & $\mathrm{mm}$ \\
\hline $\mathrm{F}_{\mathrm{D}}$ & Druckkraft & $\mathrm{N}$ \\
\hline$f_{z}$ & Vorschub pro Zahn & $\mathrm{mm} / \mathrm{U}$ \\
\hline $\mathrm{F}_{\mathrm{z}}$ & Zugkraft & $\mathrm{N}$ \\
\hline GKW & Gröditzer Kurbelwelle Wildau & \\
\hline Go & Höchstmaß & \\
\hline $\mathrm{Gu}$ & Mindestmaß & \\
\hline$h_{a}$ & $\begin{array}{l}\text { Anschnitthinterschliff (auf Zahnbreite )/ } \\
\text { Gewindebohrer }\end{array}$ & $\mathrm{mm}$ \\
\hline$h_{f}$ & $\begin{array}{l}\text { Flankenhinterschliff ( auf Zahnbreite)/ } \\
\text { Gewindebohrer }\end{array}$ & $\mathrm{mm}$ \\
\hline HM & Hartmetall & \\
\hline$h_{r}$ & $\begin{array}{l}\text { Anschnitthinterschliff (pro Teilung)/ } \\
\text { Gewindebohrer }\end{array}$ & $\mathrm{mm}$ \\
\hline HSK & Hohlschaftkegelaufnahme & \\
\hline HSS & Legierter Werkzeugstahl & \\
\hline HSSE & $\begin{array}{l}\text { Legierter Werkzeugstahl mit erhöhtem } \\
\text { Kobalt-Anteil }\end{array}$ & \\
\hline IKZ & Innere Kühlschmierstoffzufuhr, axial & \\
\hline IKZN & $\begin{array}{l}\text { Innere Kühlschmierstoffzufuhr, axial } \\
\text { mit Austritt in den Nuten }\end{array}$ & \\
\hline KHM & Vollhartmetall-Kopf & \\
\hline KSEM & KENNAMETAL Bohrer & \\
\hline$I_{1}$ & Gesamtlänge/Gewindebohrer & $\mathrm{mm}$ \\
\hline $\mathrm{I}_{2}$ & Gewindelänge & $\mathrm{mm}$ \\
\hline $\mathrm{I}_{4}$ & Anschnittlänge/Gewindebohrer & $\mathrm{mm}$ \\
\hline$I_{5}$ & Vierkantlänge/Gewindebohrer & $\mathrm{mm}$ \\
\hline $\mathrm{I}_{8}$ & Nutenlänge/Gewindebohrer & $\mathrm{mm}$ \\
\hline
\end{tabular}




\begin{tabular}{|c|c|c|}
\hline Zeichen & Beschreibung & Einheit \\
\hline$I_{E}$ & Einspannlänge/Gewindebohrer & $\mathrm{mm}$ \\
\hline$M_{t}$ & Drehmoment & $\mathrm{Nm}$ \\
\hline$N_{b}$ & Nutenbreite/Gewindebohrer & $\mathrm{mm}$ \\
\hline$n_{w}$ & Drehzahl des Werkzeuges & $\min -1$ \\
\hline P & Gewindesteigung & $\mathrm{mm}$ \\
\hline$R_{e}$ & Streckgrenze & $\mathrm{N} / \mathrm{mm}^{2}$ \\
\hline $\mathrm{Rm}$ & Zugfestigkeit & $\mathrm{N} / \mathrm{mm}^{2}$ \\
\hline $\mathrm{T}$ & Toleranz & $\mu \mathrm{m}$ \\
\hline $\mathrm{T}$ & Standzeit & $\min$ \\
\hline$t_{\text {bez }}$ & Zeit bezogen auf das Schneiden eines Gewindes & $\min$ \\
\hline TIN & Titan Nitrid & \\
\hline$v_{c}$ & Schnittgeschwindigkeit & $\mathrm{m} / \mathrm{min}$ \\
\hline VHM & Vollhartmetall & \\
\hline WZM & Werkzeugmaschine & \\
\hline y & $1 / 2$ Durchmesserverjüngung/Gewindebohrer & $\mathrm{mm}$ \\
\hline z & Zähnezahl der Bohrer & \\
\hline $\mathrm{z}_{\mathrm{b}}$ & Zahnbreite/Gewindebohrer & $\mathrm{mm}$ \\
\hline
\end{tabular}

\section{Anmerkungen}

[A1] Der Werkstoff 42CrMoNi4V ist eine Modifikation des Werkstoffes $42 \mathrm{CrMo} 4 \mathrm{~V}$. Der zusätzliche Nickelanteil ( $0,8 \%$ Ni gegenüber $0,4 \% \mathrm{Ni}$ ) sorgt für eine bessere »Durchvergütung «. So kann auf eine zusätzliche Vergütung nach der Vorbearbeitung verzichtet werden.

[A2] Ein Weldon-Schaft ist neben der Morsekegelaufnahme (MK), Steilkegelaufnahme (SK) bzw. Hohlschaftkegelaufnahme (HSK) eine Aufnahme des Werkzeuges in der Werkzeugmaschinenspindel. Bei Drehfräsmaschinen wird für die Hauptspindel, die eine HSK 160-Werkzeugaufnahme besitzt, ein zusätzlicher Adapter von HSK zu Weldon benötigt. Ideal wäre ein Bohrwerkzeug mit einer HSK 160-Aufnahme für die GKW-Drehfräsmaschinen.

\section{Literatur}

[1] Anonym (2003): VDI-Z Integrierte Produktion Special, 2, 45-46.

[2] Diethard, T. (2006): MM - Maschinenmarkt, 23, 110-113.

[3] Warurzyniak, P. (2003): Manufacturing Engineering, 3, 51-59.

[4] Rechberger, J. (2002): WB - Werkstatt und Betrieb, 12, 39-42.

[5] Balkov, V. P.; Baskov, V. M. (1999): Vestnik Masinostroenja, 1, 3537.

[6] Hammer, N.; Weinert, K. (2005): WB - Werkstatt und Betrieb, 12, 27-30.

[7] Linss, M.; Lange, D.; Watzke, R. (2005): WB - Werkstatt und Betrieb, 2005, 6, 38-42.

[8] Anonym (2008): MegaLink Precision Heft Extra Innovation, 8083.

[9] Schniering, B.; Nötzel, V. (2004): MM - Maschinenmarkt, 5, 2830.
[10] Heiler, R. (2005): WB - Werkstatt und Betrieb, 10, 54-56.

[11] Anonym (2002): MM - Maschinenmarkt, 36, 58-61.

[12] Grundlach, F. (2001): Report, 1-76.

[13] Fischer, U. (2002): Tabellenbuch Metall, Verlag Europa Lehrmittel Nourney, Vollmer GmbH \& Co, 42. Auflage.

[14] Düniß, W.; Neumann, M.; Schwartz, H. (1968): Trennen, Spanen und Abtragen, VEB Verlag Technik Berlin 1968.

[15] EMUGE-Werk Richard Glimpel GmbH \& Co KG (2004): Handbuch der Gewindetechnik und Frästechnik, Anwendungen, Tipps, Tabellen, EMUGE-FRANKEN, Lauf/Rückersdorf.

[16] EMUGE-Werk Richard Glimpel GmbH \& Co KG (2005): Gewindeschneidtechnik, Spanntechnik, Werkzeugkatalog, April 2005.

[17] Degner, W.; Lutze, H.; Smejkal, E. (2002): Spanende Formung, Theorie, Berechnung, Richtwerte, 15. Auflage, Carl Hanser Verlag München Wien.

[18] STELLRAM GmbH (2006): Werkzeug Katalog, Stellram Präzisionswerkzeuge für alle Werkzeuganwendungen, 01/2006.

[19] EMUGE-Werk Richard Glimpel GmbH \& Co KG (2005): Bedienungsanleitung für die Schnellwechselaufnahmen der Typen HF 20, HF 30 vom 01.09.2005.

[20] Preger, E.; Reindl, R. (1940): Klingelnberg, Technisches Hilfsbuch, Verlag von Julius Springer.

\section{Autoren}

Prof. Dr.-Ing. Norbert Miersch

TH Wildau [FH]

Maschinenbau/Werkzeugkonstruktion

Tel. +49 3375 508-193

norbert.miersch@tfh-wildau.de

\section{Rainer Berghaus}

Student M07

Fichtestraße 97, 15745 Wildau

Tel. +493375 219385

rainer.berghaus@tfh-wildau.de

\section{Martin Lustig}

Student M07

Buchenweg 7, 14943 Luckenwalde

Tel. +493371636656

martin.lustig@tfh-wildau.de

Dipl.-Ing. Steffen Drechsler

GKW GmbH

Leiter Kompetenzzentrum

Schmiedestraße, 15745 Wildau

Tel. +49 3375 586-334

drechsler.s@kurbelwellewildau.de 